\title{
Uma visão psiquiátrica sobre o fenômeno do crack na atualidade
}

\author{
A psychiatric view on the crack phenomenon nowadays
}

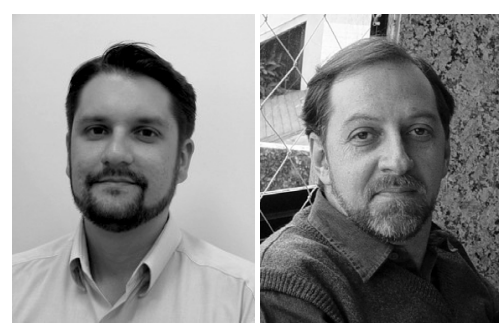

\author{
Felix Kessler ${ }^{1}$, Flavio Pechansky ${ }^{2}$
}

${ }^{1}$ Psiquiatra. Vice-diretor, Centro de Pesquisa em Álcool e Drogas, Hospital de Clínicas de Porto Alegre (HCPA), Universidade Federal do Rio Grande do Sul (UFRGS), Porto Alegre, RS. ${ }^{2}$ Psiquiatra. Diretor, Centro de Pesquisa em Álcool e Drogas, HCPA, UFRGS.

No início da década de 80 , socioetnógrafos americanos descreveram na literatura científica uma nova e potente forma de uso de cocaína - a inalação do vapor expelido da queima de pedras, manufaturadas a partir do "cozimento" da pasta básica combinada com bicarbonato de sódio. Quando queimada em um cachimbo de vidro ou outro recipiente, produzia um ruído típico de estalo, tendo sido, por isso, chamada de crack. O seu uso nesse formato permitia uma disseminação maciça da substância para o cérebro, obtendo efeitos mais estimulantes e muitíssimo prazerosos. O início de ação da droga também era rápido, porém mais fugaz, e os usuários descreviam uma "fissura" (craving, em inglês) quase incontrolável quando a estavam utilizando. Os relatos iniciais sobre os indivíduos que ousavam experimentá-la descreviam-nos como "escravos" dos seus efeitos; muitos terminavam sucumbindo devido aos danos causados ao organismo. $\mathrm{Na}$ época, as pedras eram vendidas por aproximadamente 25 dólares, segundo reportagens divulgadas nos jornais de Los Angeles e de Nova Iorque. Entretanto, mesmo após 1 ano de bombardeamento pela mídia leiga sobre esse tema, a Drug Enforcement Agency dos EUA ainda considerava essa forma de uso de cocaína como um problema menor, quando comparada à cocaína inalada ${ }^{1}$.

Alguns desses dados e relatos anedóticos foram sendo confirmados, como o grande potencial dependógeno da droga, e outros não, como a sua letalidade. De qualquer modo, no início da década de 90, várias revisões sobre o tema foram publicadas no intuito de contrapor os mitos e as evidências científicas relacionadas a essa droga, além de alertar as autoridades sobre a possibilidade de uma epidemia e sugerir formas de prevenção e tratamento da dependência e dos problemas associados a ela ${ }^{2,3}$. Contudo, de forma surpreendente, as publicações americanas foram escasseando na última década, sendo que várias dessas questões se encontram em aberto até o momento particularmente no que se refere ao tratamento dessa dependência.
A história do crack no Brasil seguiu uma trajetória semelhante, porém com um atraso de aproximadamente 10 anos em relação ao hemisfério norte. Depois da virada do milênio, vários relatos sobre esse tema foram produzidos, denotando uma preocupação cada vez maior dos profissionais da saúde e pesquisadores com o uso do crack pela população e suas conseqüências. Estudos quantitativos e qualitativos foram desenvolvidos e demonstraram, por exemplo, que o preço de uma pedra de crack no Brasil era muito menor, custando aproximadamente 2 dólares. Notou-se também que muitos dos antigos usuários de cocaína decidiram substituir o formato injetável pela via fumada. Em função do maior custo e da dificuldade de portabilidade dos cachimbos, os usuários brasileiros engenhosamente desenvolveram uma maneira de fumar através do uso de latas de alumínio furadas e com o auxílio de cinzas de cigarro, que aumentam a combustão. Além do risco continuado de queimaduras labiais, recentemente aventou-se a possibilidade de elevação dos níveis de alumínio no sangue desses usuários, o que poderia trazer mais danos ao sistema nervoso central. No que compete ao risco de exposição para o HIV, um estudo publicado em 2006 por Pechansky et $\mathrm{al}^{4}{ }^{4}$, analisando um pool de 1.449 usuários de drogas de Porto Alegre, demonstrou que o perfil dos usuários de crack é muito parecido com o dos usuários de cocaína injetável, por apresentarem baixo nível socioeconômico e maior tendência a trocar sexo por droga, o que termina se expressando por uma altíssima taxa de soroprevalência quando comparada com a de usuários de cocaína inalada.

Mais do que um dano específico ao organismo do indivíduo, está claro para a comunidade científica e leiga brasileira que o crack é uma droga de grande impacto. No momento atual, uma das questões centrais discutidas no país é a prevalência de seu consumo. Os principais estudos nessa área foram realizados pelo Centro Brasileiro de Informações sobre Drogas Psicotrópicas, sendo que apenas nos últimos levantamentos o uso de 
crack foi relacionado, corroborando estudos pontuais que sugerem haver realmente um aumento no seu consumo. O quinto levantamento, realizado entre alunos do ensino médio e fundamental das escolas públicas do Brasil, constatou que $2 \%$ dos estudantes até 18 anos usaram cocaína pelo menos uma vez na vida, e $0,7 \%$ usaram crack $^{5}$. Entretanto, é importante salientar que estudos de natureza epidemiológica obtidos em escolas têm como natural viés a obtenção de dados sobre alunos que estejam matriculados, cursando e presentes em sala de aula no dia da coleta. Por suas características peculiares, o crack não é uma droga que permita a convivência em um ambiente de ensino, e provavelmente os dados obtidos dessa forma encontramse subestimados. Do mesmo modo, o segundo levantamento domiciliar realizado em indivíduos de 12 a 65 anos nas 108 maiores cidades brasileiras e publicado em 2007 também chegou a essa mesma média de percentagem de experimentação de $c r a c k$, exceto nas regiões sul e sudeste, que costumeiramente apresentam taxas mais altas de consumo de cocaína ${ }^{6}$. Recentemente, um estudo coordenado pelo Centro de Pesquisa em Álcool e Drogas da UFRGS em cinco centros de tratamento ambulatorial e hospitalar de quatro capitais brasileiras encontrou que $39,4 \%$ dos pacientes procuraram o atendimento devido ao uso de crack. Entretanto, a avaliação do número de dependentes dessa droga na população brasileira ainda não foi estudada e exigiria métodos de aferição mais complexos.

A mídia brasileira tem relatado casos de uso de crack também nas classes média e alta, mas ainda faltam evidências científicas de que esse índice seja alarmante. Hartman \& Gollub, em 1999, analisaram reportagens publicadas em jornais americanos sobre a "epidemia do crack" e concluíram que estas eram sensacionalistas, pois não tinham embasamento científico e teriam ocasionado um desvio de foco das autoridades sobre outros problemas sociais mais relevantes ${ }^{7}$. Demonstrouse que, com a melhora da economia americana na década de 90 e a entrada de outras drogas, como opióides de alta potência e as chamadas designer drugs, houve uma diminuição de $60 \%$ no consumo de crack. Resta saber se no Brasil o fenômeno se repetirá ou se ocorrerá uma epidemia de maiores proporções. Em 2008, foi publicada uma revisão sobre o perfil dos usuários de crack brasileiro, confirmando que realmente a maior parte dos usuários ainda é jovem, de baixa renda e do sexo masculino ${ }^{8}$. O uso de crack nas classes mais altas, muitas vezes, poderia estar associado a comorbidades psiquiátricas, como os transtornos de personalidade e os de humor. Independente dos números, o que nos sensibiliza na expansão do uso de crack é a velocidade do deterioro da vida mental, orgânica e social do indivíduo. Não se pode deixar de citar o fenômeno das crianças (crack babies) intoxicadas por essa droga durante a gravidez. Sabe-se que o uso de crack durante a gestação pode desencadear abortos espontâneos, prematuridade, diminuição no crescimento do feto e outras alterações perinatais. Além disso, aqueles que nascem vivos podem apresentar retardo mental ou outros transtornos mentais e comportamentais que trarão sérias conseqüências para suas vidas ${ }^{9,10}$.

A relação entre uso de crack e mortalidade não é direta. É inegável que o índice de mortalidade entre usuários de crack seja grande, mas é importante compreender que os óbitos são mais comumente associados a elementos de tráfico, disputa entre pontos de venda/uso ou enfrentamentos com a polícia do que propriamente pelo dano causado diretamente pela droga em si. Uma coorte realizada em São Paulo por 5 anos, com 131 usuários, demonstrou em que as maiores causas de morte eram por homicídio e AIDS ${ }^{11}$. Vários estudos correlacionam o uso de crack a um aumento da agressividade, especialmente nos períodos de abstinência, e a relação entre essa agressividade e a mortalidade descrita acima é forte. As regiões onde há grande consumo dessa droga costumam apresentar índices mais altos de violência e crimes em geral.

O crack é uma droga de difícil tratamento particularmente se levarmos em consideração os modelos atualmente propostos para atendimento de drogas no Brasil. A maioria dos autores afirma que a abordagem deve ser multidisciplinar e dividida em diversas etapas através de um modelo complexo de característica biopsicossocial, enfocando especialmente as estratégias de prevenção de recaída. O uso de psicofármacos costuma auxiliar, apesar de ainda não haver uma medicação considerada eficaz para o uso de crack. Na maioria das vezes, essa abordagem inclui aspectos individuais, familiares e sociais, dirigidos aos problemas mais graves associados aos dependentes, como problemas psiquiátricos, legais e de emprego. Cabe considerar aqui que modelos preventivos de abordagem, do tipo redução de danos, parecem apresentar pouco resultado nessa população de usuários. Medidas como cachimbos descartáveis ou outras estratégias que se baseiam predominantemente na manutenção de uso seguro - bastante aceitáveis em outras modalidades de uso de substância - não apresentam eficácia comprovada em usuários de crack. Dessa forma, a estratégia que pareceria demonstrar maior resultado passa por uma estrutura de tratamento de longo prazo, que contempla uma internação inicial em ambiente psiquiátrico localizado em hospital geral e se estende para um modelo de atendimento baseado em comunidades terapêuticas fechadas ou com alto grau de intensidade de tratamento, também por longos períodos - freqüentemente de 6 meses a 1 ano. Cabe salientar que a rede familiar e social tem um papel preponderante na aderência ao tratamento, devido à 
baixa motivação dos pacientes, e no monitoramento por longo prazo durante o período de manutenção da abstinência. Os casos que não têm fácil acesso ao sistema de saúde ou não têm apoio externo costumam ter baixos índices de recuperação. Para esses casos, indica-se que a equipe de saúde procure-os ativamente, utilizando técnicas focadas na adesão ao tratamento e fornecimento de incentivos ${ }^{12}$.

Infelizmente, no Brasil, a escalada do crack coincidiu com a política de fechamento de leitos psiquiátricos, e a rede pública não tem tido capacidade de absorver toda a demanda. De acordo com João Alberto Carvalho, presidente da Associação Brasileira de Psiquiatria, o país conta com apenas 1.800 leitos psiquiátricos em hospital geral ${ }^{13}$. $\mathrm{O}$ grande desafio é o de instituir políticas preventivas para a população sob maior risco de contato com essa droga, que deveriam incluir programas sociais e alternativas ocupacionais recompensadoras. No que compete ao tratamento dos casos identificados, é mister que as orientações provenientes do Ministério da Saúde contemplem o modelo médico de assistência, devido à gravidade dos casos. É também necessário que os programas de atendimento e as políticas desenvolvidas sejam mais embasados nas evidências científicas já disponíveis sobre o tratamento das dependências químicas. Certamente que, para serem mais efetivos, esses modelos exigem uma disponibilização maior de recursos do poder público do que os tratamentos utilizados para outras doenças crônicas, o que muitas vezes gera certa resistência. Contudo, caso essas medidas não sejam implementadas brevemente, continuaremos a ver nos jornais um aumento do número de notícias e imagens estampadas sobre o descaso com o impacto dessa droga - seja sob a forma de usuários amarrados nos leitos domiciliares pelos seus familiares por pura falta de estrutura do Estado, seja nas páginas policiais.

\section{Referências}

1. Inciardi JA. Cocaine, crack and other masquerados for mama coca. In: Inciardi JA, ed. The war on drugs III - the continuing saga of the mysteries and miseries of intoxication, addiction, crime, and public policy. Boston: Allyn and Bacon; 2002. p. 129-66.

2. Cornish JW, O'Brien CP. Crack cocaine abuse: an epidemic with many public health consequences. Annu Rev Public Health. 1996;17:259-73.

3. Haasen C, Krausz M. Myths versus evidence with respect to cocaine and crack: learning from the US experience. Eur Addict Res. 2001;7(4):15960.

4. Pechansky F, Woody G, Inciardi J, Surratt H, Kessler F, Von Diemen L, et al. HIV seroprevalence among drug users: an analysis of selected variables based on 10 years of data collection in Porto Alegre, Brazil. Drug Alcohol Depend. 2006;82(suppl 1):S109-13.

5. Galduróz JC, Noto AR, Fonseca AM, Carlini EA. V Levantamento Nacional sobre o Consumo de Drogas Psicotrópicas entre Estudantes do Ensino Fundamental e Médio da Rede Pública de Ensino nas 27 Capitais Brasileiras. São Paulo: CEBRID/UNIFESP; 2004.

6. Carlini EA, Galduróz JC, Noto AR, Carlini CM, Oliveira LG, Nappo SA, et al. II Levantamento Domiciliar sobre o Uso de Drogas Psicotrópicas no Brasil: estudo envolvendo as 108 maiores cidades do país, 2005. São Paulo: CEBRID/UNIFESP; 2007.

7. Hartman DM, Golub A. The social construction of the crack epidemic in the print media. J Psychoactive Drugs. 1999;31(4):423-33.

8. Duailib LB, Ribeiro M, Laranjeira R. Profile of cocaine and crack users in Brazil. Cad Saude Publica. 2008;24(supl 4):545-57.

9. Litt J, McNeil M. Biological markers and social differentiation: crack babies and the construction of the dangerous mother. Health Care Women Int. 1997;18(1):31-41.

10. Lyons P, Rittner B. The construction of the crack babies phenomenon as a social problem. Am J Orthopsychiatry. 1998;68(2):313-20.

11. Ribeiro M, Dunn J, Sesso R, Dias AC, Laranjeira R. Causes of death among crack cocaine users. Rev Bras Psiquiatr. 2006;28(3):196-202.

12. Henskens R, Garretsen H, Bongers I, Van Dijk A, Sturmans F. Effectiveness of an outreach treatment program for inner city crack abusers: compliance, outcome, and client satisfaction. Subst Use Misuse. 2008;43(10):1464-75.

13. Carvalho JA. Pela inclusão dos pacientes mentais na rede geral de saúde [editorial]. Psiquiatria Hoje. 2008;3(4):3. 